\section{EFECTO DE LA DENSIDAD CELULAR DE INOCULACIÓN EN EL CRECIMIENTO DE Chlorella vulgaris CLV2 CULTIVADA BAJO CONDICIONES MIXOTRÓFICAS}

\section{EFFECT OF INOCULATION CELLULAR DENSITY ON Chlorella vulgaris CLV2 GROWTH CULTIVATED UNDER MIXOTROPHIC CONDITIONS}

\author{
Heberto A. Rodas-Gaitán, Humberto Rodríguez- \\ Fuentes ${ }^{\star}$, Gerardo Flores-Mendiola, Juan A. \\ Vidales-Contreras, Juana Aranda-Ruiz y Alejandro I. \\ Luna-Maldonado
}

Subdirección de Estudios de Posgrado, Facultad de Agronomía, Universidad Autónoma de Nuevo León, Campus de Ciencias Agropecuarias. Av. Francisco Villa s/n, col. Ex Hacienda El Canadá. 66050, Municipio de General Escobedo, Nuevo León, México.

${ }^{\star}$ Autor para correspondencia (hrodrigu10@yahoo.com.mx)

\section{RESUMEN}

El uso de microalgas para el aprovechamiento de biomoléculas (proteínas, carbohidratos y lípidos) ha tomado auge en los últimos años. Entre ellas se encuentra Chlorella vulgaris, microalga unicelular verde motil de forma esférica del Filo Chlorophyta. En este trabajo se evaluaron tres densidades de inoculación de Chlorella vulgaris CLV2 y su efecto en el crecimiento bajo condiciones mixotróficas;los datos se ajustaron a modelos sigmoidales en cada densidad. En el Tratamiento 1 (1 x $10^{6}$ células $\mathrm{mL}^{-1}$ ) el mejor modelo tuvo una $\mathrm{R}^{2}$ de 0.85 y un tiempo de generación de $59 \mathrm{~h}$. En el Tratamiento $2\left(2 \times 10^{6}\right.$ células $\left.\mathrm{mL}^{-1}\right)$ el mejor modelo tuvo una $\mathrm{R}^{2}$ de 0.89 y un tiempo de generación de $23.5 \mathrm{~h}$. En el Tratamiento $3\left(5 \times 10^{6}\right.$ células $\left.\mathrm{mL}^{-1}\right)$ el modelo tuvo una $\mathrm{R}^{2}$ de 0.92 y un tiempo de generación de $7.5 \mathrm{~h}$. El Tratamiento 3 que consistió en la mayor densidad celular de inoculación, presentó el mayor crecimiento y el menor tiempo de generación, porque redujo el tiempo en alcanzar cada fase de crecimiento y sin presencia de microorganismos contaminantes.

Palabras clave: Microalga, fases de crecimiento, curva de crecimiento, tiempo de generación.

\section{SUMMARY}

The use of microalgae to produce biomolecules (proteins, carbohydrates and lipids) has increased in the last years. One of them is Chlorella vulgaris, a green motile unicellular microalga, of spherical form in the Chlorophyta Phylum. In this research three cellular inoculation densities were evaluated regarding their effect on algae growth under mixotrophic conditions, and sigmoidal models were adjusted to the obtained data in each density. In Treatment $1\left(1 \times 10^{6}\right.$ cells $\left.\mathrm{mL}^{-1}\right)$ the best model had an $R^{2}$ of 0.85 and a generation time of $59 \mathrm{~h}$. In Treatment 2 $\left(2 \times 10^{6}\right.$ cells $\left.\mathrm{mL}^{-1}\right)$ the best model had an $\mathrm{R}^{2}$ of 0.89 and a generation time of $23.5 \mathrm{~h}$. In Treatment $3\left(5 \times 10^{6}\right.$ cells $\left.\mathrm{mL}^{-1}\right)$ the model had an $\mathrm{R}^{2}$ of 0.92 and a generation time of $7.5 \mathrm{~h}$. Treatment 3 , with the highest cellular inoculation density, showed the highest growth and shortest generation time; thus it decreased the time to reach each growth phase, and contaminating microorganisms were absent.
Index words: Microalgae, growth phases, growth curve, generation time.

\section{INTRODUCCIÓN}

El cultivo de Chlorella vulgaris presenta uso potencial en producción de biodiesel, descontaminación de aguas residuales y gases de chimenea, y en nutrición humana y animal, por lo cual se encuentra en el punto central de diversas investigaciones. Sin embargo, existen limitaciones que se deben superar para la producción de biomasa a gran escala y factores que se deben controlar para hacer un manejo eficaz del cultivo para la obtención de biomasa seca (BS); dentro de estos últimos se puede mencionar: densidad celular de inoculación, cantidad y calidad de luz, temperatura, nivel de agitación, así como $\mathrm{pH}$, fuente de carbono, concentración y composición de nutrientes y condiciones estériles de la solución nutritiva. Además, para cada cepa específica es imprescindible conocer el manejo adecuado de los factores anteriormente mencionados, y de esta forma optimizar la producción de biomasa (Ogbonna et al., 1999; Andersen, 2005).

El tiempo de cosecha, intervalo entre muestreos y periodo adecuado para renovación del cultivo madre durante la fase exponencial es contradictorio y depende de las condiciones de crecimiento y densidad celular de inoculación (Bertoldi et al., 2006; Bertoldi et al., 2009; Hongjin y Guangce, 2009; Liang et al., 2009). El conocimiento de la dinámica de crecimiento de microorganismos (tasa de inoculación, densidad celular en el tiempo, máxima tasa de crecimiento, tiempo de generación y producción de biomasa, entre otros) y su manipulación, es indispensable para generar un sistema viable de producción tanto técnica como económicamente. El objetivo del presente trabajo fue evaluar tres densidades celulares de inoculación en Chlorella vulgaris CLV2 y su efecto en el crecimiento bajo condiciones mixotróficas.

\section{MATERIALES Y MÉTODOS}

El experimento se llevó a cabo en el Laboratorio de Biotecnología Microbiana de la Facultad de Agronomía de la Universidad Autónoma de Nuevo León, ubicado en el Municipio de Escobedo, Nuevo León, México. La cepa de Chlorella vulgaris CLV2 fue adquirida en el Departamento de Acuicultura, del Centro de Investigación Científica y de Educación Superior de Ensenada, Baja California Norte, México.

\section{Manejo del experimento y toma de datos}

Se evaluaron tres densidades celulares de inoculación: $1 \times 10^{6}$ células $\mathrm{mL}^{-1}$ (Tratamiento 1 ), $2 \times 10^{6}$ células $\mathrm{mL}^{-1}$ (Tratamiento 2) y $5 \times 10^{6}$ células $\mathrm{mL}^{-1}$ (Tratamiento 3 ). El medio de cultivo utilizado fue BBM (Bold's Basal Medium) (Bertoldi et al., 2006; Bertoldi et al., 2009; Hongjin y Guangce, 2009). El pH inicial del medio de cultivo fue ajustado a 6.8. La fuente de carbono utilizada fue glucosa 
a $1 \%$. Los cultivos fueron mantenidos en incubadora (Incubator-Shaker ${ }^{\circledR}$ Model 3525; Lab-Line Instruments Inc., India) bajo condiciones de cultivo en lote. La velocidad de agitación fue de $125 \mathrm{rpm}$. La fuente de energía lumínica fue provista por dos lámparas fluorescentes tipo "Cool White" de $50 \mathrm{~W}$. La temperatura a la cual se mantuvo el cultivo fue de $28 \pm 2{ }^{\circ} \mathrm{C}$. De acuerdo con las fuentes de carbono y de energía empleadas, el cultivo del alga se hizo bajo condiciones mixotróficas (Liang et al., 2009).

Los tres tratamientos se reprodujeron en frascos Erlenmeyer de $0.5 \mathrm{~L}$ de capacidad, con un volumen de cultivo $367.5 \mathrm{~mL}$. La densidad celular se cuantificó con un hemocitómetro o cámara de Neubauer y con un microscopio (Colomé et al., 1986). Previamente se evaluó la duración de cada fase de crecimiento en los tres tratamientos, con el objetivo de establecer los intervalos de muestreos para cada uno (datos no reportados). Para el Tratamiento 1 los muestreos se hicieron cada $24 \mathrm{~h}$ (durante $168 \mathrm{~h}$ ); para el Tratamiento 2 fueron cada $12 \mathrm{~h}$ (durante $72 \mathrm{~h}$ ); y para el Tratamiento 3 se hicieron a las $12,24,26,28,30,32,34,36$, 46 y 70 h. Se colectó $2 \mathrm{~mL}$ del cultivo en cada repetición del muestreo, y se consideraron cuatro repeticiones por muestreo. Cada tratamiento fue repetido tres veces en total. De acuerdo con el procedimiento descrito por Maier et al. (2000), el tiempo de generación de Chlorella vulgaris CLV2 fue calculado a partir de los datos correspondientes al inicio y final de la fase exponencial. Los análisis de varianza se hicieron conforme a un diseño experimental completamente al azar, con los muestreos de las 24 y 48 h. Los valores medios se separaron con la prueba de rango múltiple de Tukey $(\mathrm{P} \leq 0.05)$, mediante el programa SPSS17.0. Los resultados obtenidos de densidad celular medidos a través del tiempo fueron graficados en busca del modelo sigmoidal de mejor ajuste, mediante el programa SigmaPlot $10.0^{\mathrm{TM}}$, donde se tomó como criterio de decisión el mayor valor del coeficiente de determinación $\left(\mathrm{R}^{2}\right)$.

\section{RESULTADOS Y DISCUSIÓN}

Los promedios y sus desviaciones estándar (DE) del conteo celular se presentan en el Cuadro 1. En general se observó una mayor $\mathrm{DE}$ en aquellos muestreos con la mayor densidad celular, que correspondió con las fases exponencial y estacionaria del Tratamiento 3; esto se atribuye a que en éstos hubo mayor dificultad para hacer el conteo en la cámara de Neubauer, ya que al aumentar el número de células, éstas se agruparon en forma de colonias o se traslaparon. También Bertoldi et al. (2009) reportaron que los conteos celulares realizados durante las últimas fases de crecimiento en el cultivo de Chlorella vulgaris (densidad celular cercana a $10 \times 10^{6}$ células $\mathrm{mL}^{-1}$ ) presentaron una mayor $\mathrm{DE}$. Si bien un grupo de investigadores (Bertoldi et al., 2006; Bertoldi et al., 2009) han usado la cámara de Neubauer para cuantificar el crecimiento celular de Chlorella vulgaris, aquí se evidencia que este método presenta errores que pueden afectar los análisis estadísticos en algunos casos, por lo que sería conveniente optar por determinar BS, medir turbidez, o algún otro método.

Los Tratamientos 1, 2 y 3 se ajustaron a modelos sigmoidales (Cuadro 2), con tiempos de generación y coeficientes de determinación $\left(\mathrm{R}^{2}\right)$ de: 59 h y $0.85,23.5$ h y $0.89,7.5$ h y 0.92 , respectivamente. La Figura 1 muestra que a medida que la densidad de inoculación fue menor, la fase exponencial se alcanzó en mayor tiempo. Esto concuerda con los reportes de Maier et al. (2000) y Sarma et al. (2008).

En el Tratamiento 1 se registró un crecimiento lento hasta la fase de establecimiento (7 d). En el Tratamiento 2 la fase de establecimiento duró aproximadamente $18 \mathrm{~h}$ y la fase exponencial se alcanzó a las $41 \mathrm{~h}$. En el Tratamiento 3 la fase de establecimiento duró aproximadamente $18 \mathrm{~h}$ y la fase exponencial se alcanzó a las $30 \mathrm{~h}$. En los Tratamientos 1 y 2 no solamente se prolongaron los tiempos necesarios para alcanzar cada fase de crecimiento, sino también fue afectado el crecimiento celular al mostrar una disminución con respecto al Tratamiento 3 (Figura 1). Lo anterior fue debido a la presencia de microorganismos contaminantes y competidores presentes en los cultivos de los Tratamientos 1 y 2 , los cuales fueron observados al microscopio (100X), los cuales disminuyeron la coloración verde intensa característica de Chlorella vulgaris CLV2 (datos no reportados). Por el contrario, en el Tratamiento 3 se alcanzó el máximo crecimiento ( $1.66 \times 10^{7}$ células $\mathrm{mL}^{-1}$ en $36 \mathrm{~h}$ ), sin haber detectado microorganismos contaminantes al microscopio (100 X) y se mantuvo la coloración verde intensa característica de la microalga. Esto concuerda con lo reportado por Sarma et al. (2008), quienes mencionaron que al aumentar la densidad celular de inoculación de Chlorella vulgaris de 2 x $10^{5}$ a $8 \times 10^{5}$ células $\mathrm{mL}^{-1}$, también aumentó la dominancia sobre otros microorganismos competitivos.

Algunos investigadores usaron densidades celulares de inoculación relativamente bajas $\left(<1 \times 10^{6}\right.$ células $\left.\mathrm{mL}^{-1}\right)$, y alcanzaron la fase exponencial en periodos largos $(>5 \mathrm{~d})$; tal es el caso de Bertoldi et al. (2009), quienes inocularon a una densidad de $1.16 \times 10^{5}$ células $\mathrm{mL}^{-1}$ y la máxima densidad obtenida a los $7 \mathrm{~d}$ fue de $10.6 \times 10^{6}$ células $\mathrm{mL}^{-1}$. Se han reportado casos de producción de Chlorella vulgaris a nivel de escalamiento en los que se ha inoculado a una densidad de $1 \times 10^{5}$ células $\mathrm{mL}^{-1}$, y el máximo crecimiento se ha alcanzado a los $38 \mathrm{~d}$ con una producción de $1.09 \times 10^{7}$ células $\mathrm{mL}^{-1}$, equivalente a $4.5 \mathrm{~g} \mathrm{~L}^{-1}$ de biomasa (Armendáriz et al., 2010). Según Drapcho et al. (2008), un proyecto viable de producción microalgal deberá generar biomasa en cortos periodos de tiempo. Además, es necesario considerar que aunque algunos autores reportan producciones altas de BS ( $2 \mathrm{~g} \mathrm{~L}^{-1}$ bajo condiciones mixotróficas), ésta es alcanzada en periodos largos $(6 \mathrm{~d})$ y requieren de equipo especializado (Ogbonna et al., 1999; Liang et al., 2009).

Una vez aplicados los tratamientos, éstos fueron manejados bajo condiciones mixotróficas, con glucosa como fuente de carbono. Según Haass y Tanner (1974), Martínez 
Cuadro 1. Densidades celular promedio (células $\mathrm{mL}^{-1}$ ) y desviación estándar (DE) de Chlorella vulgaris $C L V 2$, por muestreo ${ }^{\dagger}$ y por tratamiento.

\begin{tabular}{ccccccc}
\hline & Tratamiento $1^{\dagger \dagger}$ & & \multicolumn{3}{c}{ Tratamiento 2 } \\
\cline { 1 - 2 } \cline { 5 - 6 } Muestreo $(\mathrm{h})$ & Densidad celular & DE & & Muestreo (h) & Densidad celular & DE \\
\hline 0 & $1.00 \times 10^{6}$ & 0 & & 0 & $2.00 \times 10^{6}$ & 0 \\
24 & $3.90 \times 10^{6}$ & $8.55 \times 10^{5}$ & & 12 & $2.60 \times 10^{6}$ & $9.53 \times 10^{5}$ \\
48 & $2.25 \times 10^{6}$ & $8.49 \times 10^{5}$ & & 24 & $3.50 \times 10^{6}$ & $8.91 \times 10^{5}$ \\
72 & $2.33 \times 10^{6}$ & $2.23 \times 10^{6}$ & & 36 & $4.39 \times 10^{6}$ & $1.49 \times 10^{6}$ \\
96 & $4.30 \times 10^{6}$ & $3.25 \times 10^{6}$ & & 48 & $6.11 \times 10^{6}$ & $9.23 \times 10^{5}$ \\
120 & $9.58 \times 10^{6}$ & $5.62 \times 10^{6}$ & & 60 & $6.63 \times 10^{6}$ & $1.94 \times 10^{6}$ \\
144 & $6.70 \times 10^{6}$ & $3.32 \times 10^{6}$ & & 72 & $5.09 \times 10^{6}$ & $4.67 \times 10^{5}$ \\
168 & $1.08 \times 10^{7}$ & $4.24 \times 10^{5}$ & & & &
\end{tabular}

Tratamiento $3^{\text {ฯा }}$

\begin{tabular}{ccl}
\hline Muestreo $(\mathrm{h})$ & Densidad celular & \multicolumn{1}{c}{ DE } \\
\hline 0 & $5.00 \times 10^{6}$ & 0 \\
12 & $6.89 \times 10^{6}$ & $2.08 \times 10^{6}$ \\
24 & $1.05 \times 10^{7}$ & $2.89 \times 10^{6}$ \\
26 & $1.36 \times 10^{7}$ & $3.17 \times 10^{6}$ \\
28 & $1.21 \times 10^{7}$ & $1.58 \times 10^{6}$ \\
30 & $1.54 \times 10^{7}$ & $3.04 \times 10^{6}$ \\
32 & $1.68 \times 10^{7}$ & $1.59 \times 10^{6}$ \\
34 & $1.72 \times 10^{7}$ & $1.99 \times 10^{6}$ \\
36 & $1.66 \times 10^{7}$ & $4.07 \times 10^{6}$ \\
46 & $1.60 \times 10^{7}$ & $3.23 \times 10^{6}$ \\
70 & $1.43 \times 10^{7}$ & $1.41 \times 10^{5}$
\end{tabular}

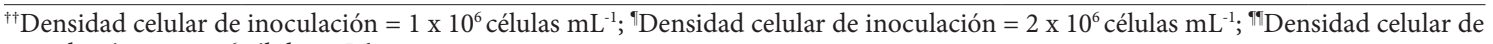
inoculación $=5 \times 10^{6}$ células $\mathrm{mL}^{-1}$.

Cuadro 2. Comparación de tratamientos por densidad celular a las 24 y $48 \mathrm{~h}$ de cultivo.

\begin{tabular}{ccclcc}
\hline Tratamiento & Media $^{\dagger}(24 \mathrm{~h})$ & $\operatorname{Media}^{\dagger}(48 \mathrm{~h})$ & Ecuación $^{2}$ & $\mathrm{R}^{2}$ \\
\hline $1^{\dagger \dagger}$ & $3.90 \times 10^{6} \mathrm{~b}$ & $2.25 \times 10^{6} \mathrm{c}$ & $\mathrm{y}=11431528.9 /\left(1+\mathrm{e}^{\wedge}(-(\mathrm{x}-103.4) / 32.5)\right)$ & 0.85 \\
$2^{\text {ศ }}$ & $3.50 \times 10^{6} \mathrm{~b}$ & $6.11 \times 10^{6} \mathrm{~b}$ & $\mathrm{y}=2229346.5+3787767.3 \mathrm{e}^{\wedge}\left(-\mathrm{e}^{\wedge}(-(\mathrm{x}-25.4) / 10.6)\right)$ & 0.89 \\
$3^{\text {ศศ }}$ & $1.05 \times 10^{7} \mathrm{a}$ & $1.61 \times 10^{7} \mathrm{a}$ & $\mathrm{y}=5959061.9+10174169.7 \mathrm{e}^{\wedge}\left(-\mathrm{e}^{\wedge}(-(\mathrm{x}-23.4) / 2.9)\right)$ & 0.92 &
\end{tabular}

Medias con letras iguales no son estadísticamente diferentes (Tukey, 0.05).

${ }^{\dagger} \mathrm{n}=12$; ${ }^{\dagger}$ Densidad celular de inoculación $=1 \times 10^{6}$ células $\mathrm{mL}^{-1}$; ${ }^{4}$ Densidad celular de inoculación $=2 \times 10^{6}$ células $\mathrm{mL}^{-1}$; "Densidad celular de inoculación $=5 \times 10^{6}$ células $\mathrm{mL}^{-1}$.

y Orús (1991), Xiong et al. (2008), Hongjin y Guangce (2009) y Liang et al. (2009), la glucosa como fuente de carbono induce la absorción de hexosas e incrementa la densidad celular y biomasa en menor tiempo que otras fuentes de carbono orgánicas e inorgánicas.

El análisis de varianza evidenció diferencias significativas $(\mathrm{P} \leq 0.05)$ entre tratamientos, tanto a las 24 como a las $48 \mathrm{~h}$ (Cuadro 2). A las $24 \mathrm{~h}$, el crecimiento del Tratamiento 3 su- peró al de los Tratamientos 1 y 2, y entre estos dos últimos no hubo diferencia significativa, quizás debido a su lento crecimiento inicial. A las 48 h, el Tratamiento 3 presentó el mayor crecimiento, seguido por el Tratamiento 2 y finalmente del Tratamiento 1.

Para la cepa Chlorella vulgaris CLV2, el aumento en la densidad celular de inoculación acortó todas las fases de crecimiento, así como el tiempo de generación. 


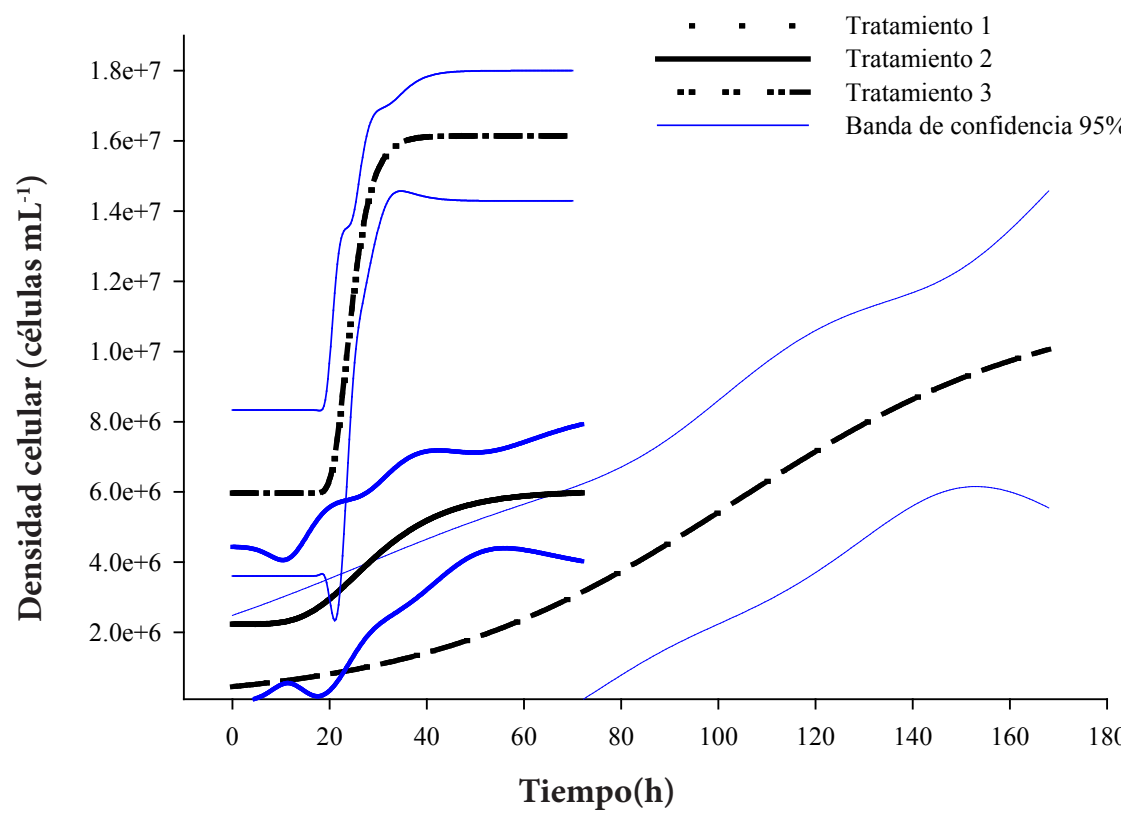

Figura 1. Modelos sigmoidales ajustados a los datos de crecimiento celular de Chlorella vulgaris CLV2. (Procesados con SigmaPlot10.0 ${ }^{\mathrm{MR}}$ ).

\section{CONCLUSIONES}

Los datos de densidad celular de los Tratamientos 1, 2 y 3 se ajustaron a modelos sigmoidales. La inoculación en cultivos de Chlorella vulgaris CLV2 a densidades de $5 \times 10^{6}$ células $\mathrm{mL}^{-1}$ (Tratamiento 3 ) mostraron los mejores resultados al alcanzar el máximo incremento a las $36 \mathrm{~h}$ posteriores a la inoculación, con un valor medio de $1.66 \times 10^{7}$ células $\mathrm{mL}^{-1}$, con un tiempo de generación de $7.5 \mathrm{~h}$.

\section{BIBLIOGRAFÍA}

Andersen R A (2005) Algal Culturing Techniques. Academy Press. USA. $596 \mathrm{p}$.

Armendáriz J F, A Posadas, M A Amezcua-Allieri (2010) Evaluación del crecimiento de la microalga Chlorella vulgaris a nivel de escalamiento para la producción de biomasa. In: Mem. II Congreso Latinoamericano de Biotecnología Ambiental y Algal. G C Sandoval F, E J Olguín P, G Sánchez G (eds). Cancún, México. 5-9 de diciembre 2010. pp:262-265.

Bertoldi F C, E Sant 'Anna, M V da Costa Braga, J L Barcelos-Olivieira (2006) Lipids, fatty acids composition and carotenoids of Chlorella vulgaris cultivated in hydroponic wastewater. Grasas y Aceites 27:270-274.

Bertoldi F C, E Sant'Anna, J L Barcelos-Olivieira (2009) Chlorella vulgaris cultivated in hydroponic wastewater. Acta Hort. 843:203210.

Colomé J, R Cano, A Kubinski, D Grady (1986) Laboratory Exercises in Microbiology. West Publishing Company. USA. 283 p.
Drapcho C M, N P Nhuan, T H Walker (2008) Biofuels Engineering Process Technology. McGraw-Hill. USA. 371 p.

Haass D, W Tanner (1974) Regulation of hexose transport in Chlorella vulgaris. Plant Physiol. 53:14-20.

Hongjin Q, W Guangce (2009) Effect of carbon source on growth and lipid accumulation in Chlorella sorokiniana GXNN01. Chin. J. Oceanol. Limnol. 27:762-768.

Liang Y, N Sarkany, Y Cui (2009) Biomass and lipid productivities of Chlorella vulgaris under autotrophic, heterotrophic and mixotrophic growth conditions. Biotechnol. Lett. 31:1043-1049.

Maier R, I Pepper, C Gerba (2000) Environmental Microbiology. Academic Press. USA. 585 p.

Martínez F, M I Orús (1991) Interactions between glucose and inorganic carbon metabolism in Chlorella vulgaris strain UAM 101. Plant Physiol. 95:1150-1155.

Ogbonna J C, T Soejima, H Tanaka (1999) Development of efficient large-scale photobioreactors, a key factor for practical production of biohydrogen. BioHydrogen VII:329-343.

Sarma S S, J L Franco, S Nandini (2008) Effect of algal food (Chlorella vulgaris) concentration and inoculation density on the competition among three planktonic brachionidae (Rotifera: Monogononta). Hidrobiológica 18:123-132.

SigmaPlot (Scientific Data Analysis and Graphing Software) Systat Software Inc. (version10.0). Disponible en: http://www.sigmaplot. com.

SPSS, Statistical Package for the Social Sciences IBM incorporated. PASW (Predictive Analytics SoftWare). (Version 17.0). Disponible en: http://www-01.ibm.com/software/analytics/spss/

Xiong W, X Li, J Xiang, Q Wu (2008) High-density fermentation of microalga Chlorella protothecoides in bioreactor for microbio-diesel production. Appl. Microbiol. Biotechnol. 78:29-36. 